\title{
On Distributions of One Class of Random Sums and their Applications
}

\author{
Ivan Matsak $^{1}$, Mikhail Moklyachuk ${ }^{2, *}$ \\ ${ }^{1}$ Department of Operation Research, Taras Shevchenko National University of Kyiv, Ukraine \\ ${ }^{2}$ Department of Probability Theory, Statistics and Actuarial Mathematics, Taras Shevchenko National University of Kyiv, Ukraine
}

\begin{abstract}
We propose results of the investigation of properties of the random sums of random variables. We consider the case, where the number of summands is the first moment of an event occurrence. An integral equation is presented that determines distributions of random sums. With the help of the obtained results we analyse the distribution function of the time during which the Geiger-Muller counter will not lose any particles, the distribution function of the busy period of a redundant system with renewal, and the distribution function of the sojourn times of a single-server queueing system.
\end{abstract}

Keywords Random sums, queueing, reliability, redundant system, renewal

AMS 2010 subject classifications. Primary: 60E05, 60K25, Secondary: 90B22

DOI: $10.19139 /$ soic-2310-5070-698

\section{Introduction}

Let $\zeta_{i}, i \geq 1$, be independent identically distributed (i.i.d.) random variables, Let $\nu$ be a discrete random variable that takes integer positive values. Consider the random sum

$$
S_{\nu}=\sum_{i=1}^{\nu} \zeta_{i}
$$

In many applied problems of reliability theory, queueing theory, some statistical problems of physics and biology, there is a need to find the distribution of the random variable $S_{\nu}$ or at least its main characteristics. If the random variable $\nu$ is a Markov moment, then the most famous result here is Wald's identity

$$
\mathbf{E} S_{\nu}=\mathbf{E} \nu \mathbf{E} \zeta_{1}
$$

(see [2], [3], [5], [16], [23], [13], [14], [20], [21] where you can also find a number of related results and applications).

Similar problems that arise in the mathematical theory of reliability, were discussed on lectures delivered by B. V. Gnedenko at Taras Shevchenko National University of Kyiv, back in 80-th years of the last century, as well as in a number of his works (see, for example, his preface to the book [19]).

The case where the random variable $\nu$ and the sequence $\left(\zeta_{i}\right)$ are independent, is studied in details (see [5], [19]). Unfortunately in practice quite often the random variable $\nu$ and the sequence $\left(\zeta_{i}\right)$ are dependent, which significantly complicates the problems.

\footnotetext{
*Correspondence to: Mikhail Moklyachuk (Email: Moklyachuk@gmail.com). Department of Probability Theory, Statistics and Actuarial Mathematics, Taras Shevchenko National University of Kyiv, Volodymyrska 64 Str., Kyiv 01601, Ukraine.
}

ISSN 2310-5070 (online) ISSN 2311-004X (print)

Copyright (C) 2020 International Academic Press 
In this paper we consider one important case of such dependence, where $\nu$ is the first moment of an event occurrence. In fact ours analysis is a definite generalization of the methods developed by B. V. Gnedenko in his works [9], [10] when studying the problem of reliability of redundant systems with renewal.

\section{Distribution of random sums. Main proposition}

Let $\zeta$ and $\epsilon$ be random variables such that

$$
\begin{gathered}
\mathbf{P}(\epsilon=1)=q, \quad \mathbf{P}(\epsilon=0)=1-q, \quad 0<q<1, \\
\mathbf{P}(\zeta \geq 0)=1, \quad \mathbf{P}(\zeta=0)<1 .
\end{gathered}
$$

In general case the random variables $\zeta$ and $\epsilon$ depend on each other.

Consider the sequence $\left(\zeta_{n}, \epsilon_{n}\right)$ of independent copies of $(\zeta, \epsilon)$. Define a random variable $\nu$ in the following way

$$
\nu=\min \left(n \geq 1: \quad \epsilon_{n}=1\right) .
$$

It is well known, that the random variable $\nu$ has the geometrical distribution

$$
\mathbf{P}(\nu=n)=q(1-q)^{n-1}, n \geq 1,
$$

and

$$
\mathbf{E} \nu=\frac{1}{q}, \quad \mathbf{D} \nu=\frac{1-q}{q^{2}} .
$$

Consider the random variable $S_{\nu}$ determined by equality (1).

We introduce the following notations

$$
\begin{aligned}
F_{\zeta, 0}(t) & =\mathbf{P}(\zeta<t, \epsilon=0), \quad F_{\zeta, 1}(t)=\mathbf{P}(\zeta<t, \epsilon=1), \\
F_{\zeta}(t) & =F_{\zeta, 0}(t)+F_{\zeta, 1}(t)=\mathbf{P}(\zeta<t), \\
F_{S}(t) & =\mathbf{P}\left(S_{\nu}<t\right), \quad P_{S}(t)=1-F_{S}(t) .
\end{aligned}
$$

Let

$$
\begin{gathered}
\psi(z)=\int_{0}^{\infty} \exp (-z t) d F_{\zeta}(t), \quad \psi_{0}(z)=\int_{0}^{\infty} \exp (-z t) d F_{\zeta, 0}(t), \\
\varphi(z)=\int_{0}^{\infty} \exp (-z t) d F_{S}(t) .
\end{gathered}
$$

be the Laplace transforms of the corresponding distribution functions.

Introduce the notations

$$
a=\mathbf{E} \zeta, \quad \sigma^{2}=\mathbf{D} \zeta, \quad a_{0}=\mathbf{E} \zeta I(\epsilon=0)=\int_{0}^{\infty} t d F_{\zeta, 0}(t),
$$

where $I(A)$ is the indicator of the event $A$.

Proposition 2.1. (i). The function $P_{S}(t)$ satisfies the integral equation

$$
P_{S}(t)=1-F_{\zeta}(t)+\int_{0}^{t} P_{S}(t-x) d F_{\zeta, 0}(x) .
$$

(ii). The Laplace transform $\varphi(z)$ satisfies the equality

$$
\varphi(z)=\frac{\psi(z)-\psi_{0}(z)}{1-\psi_{0}(z)}
$$


(iii). Under the condition $\sigma^{2}<\infty$ we have the following relations

$$
\begin{gathered}
\mathbf{E} S_{\nu}=\frac{a}{q}, \\
\mathbf{D} S_{\nu}=\frac{\sigma^{2}}{q}+\frac{a^{2}(q-1)+2 a a_{0}}{q^{2}} .
\end{gathered}
$$

Proof

(i). Let

$$
\begin{gathered}
A=\left\{S_{\nu} \geq t\right\}, \quad A_{1}=\left\{\zeta_{1} \geq t\right\} \\
A_{2}=\left\{\zeta_{1}<t, \quad \epsilon_{1}=0, \quad \sum_{i=2}^{\nu} \zeta_{i} \geq t-\zeta_{1}\right\} .
\end{gathered}
$$

Then

$$
A=A_{1} \bigcup A_{2}, \quad A_{1} \bigcap A_{2}=\emptyset,
$$

and

$$
\mathbf{P}(A)=\mathbf{P}\left(A_{1}\right)+\mathbf{P}\left(A_{2}\right) .
$$

The first term in equality (9) is quite simple

$$
\mathbf{P}\left(A_{1}\right)=1-F_{\zeta}(t)
$$

Let $\nu^{\prime}=\min \left(n \geq 2: \epsilon_{n}=1\right)$ and we know that event $\left\{\epsilon_{1}=0\right\}$ occurred. Then $\nu=\nu^{\prime}$. The random variable $\nu^{\prime}$ does not depend on the random variables $\epsilon_{1}$ and $\zeta_{1}$. Therefore, the relation

$$
\begin{gathered}
\mathbf{P}\left(\sum_{i=2}^{\nu} \zeta_{i} \geq y / \epsilon_{1}=0, \zeta_{1}=x\right)=\mathbf{P}\left(\sum_{i=2}^{\nu^{\prime}} \zeta_{i} \geq y / \epsilon_{1}=0, \zeta_{1}=x\right)=\mathbf{P}\left(\sum_{i=2}^{\nu^{\prime}} \zeta_{i} \geq y\right) \\
=\mathbf{P}\left(\sum_{i=1}^{\nu} \zeta_{i} \geq y\right)=P_{S}(y) .
\end{gathered}
$$

holds true and we have

$$
\begin{aligned}
\mathbf{P}\left(A_{2}\right) & =\int_{0}^{t} \mathbf{P}\left(\sum_{i=2}^{\nu} \zeta_{i} \geq t-x / \epsilon_{1}=0, \zeta_{1}=x\right) d F_{\zeta, 0}(x) \\
& =\int_{0}^{t} P_{S}(t-x) d F_{\zeta, 0}(x) .
\end{aligned}
$$

Equalities (9) - (11) together give equality (5).

(ii). Equality (6) follows from (5), if we use properties of the Laplace transform. Really, in terms of the Laplace transform equation (5) may be written in the form

$$
\frac{1}{z}-\frac{\varphi(z)}{z}=\frac{1}{z}-\frac{\psi(z)}{z}+\left(\frac{\psi_{0}(z)}{z}-\frac{\varphi(z) \psi_{0}(z)}{z}\right)
$$

which gives (6) (similar reasoning can be found in [11], p.331).

(iii). Equality (7) is a particular case of Wald's identity (see also relation (3)). 
Now prove (8). Differentiating equation (6) twice we get

$$
\begin{aligned}
\varphi^{\prime \prime}(z) & =\frac{\left(\psi^{\prime \prime}(z)-\psi_{0}^{\prime \prime}(z)\right)\left(1-\psi_{0}(z)\right)+\psi_{0}^{\prime \prime}(z)\left(\psi(z)-\psi_{0}(z)\right)}{\left(1-\psi_{0}(z)\right)^{2}} \\
& +\frac{2\left(1-\psi_{0}(z)\right) \psi_{0}^{\prime}(z)\left(\left(\psi^{\prime}(z)-\psi_{0}^{\prime}(z)\right)\left(1-\psi_{0}(z)\right)+\psi_{0}^{\prime}(z)\left(\psi(z)-\psi_{0}(z)\right)\right)}{\left(1-\psi_{0}(z)\right)^{4}} .
\end{aligned}
$$

Take here $z=0$ and use the know equalities

$$
\psi_{0}(0)=\mathbf{P}(\epsilon=0)=1-q, \quad \psi^{\prime}(0)=-\mathbf{E} \zeta=-a, \quad \psi_{0}^{\prime}(0)=-a_{0},
$$

we will have

$$
\varphi^{\prime \prime}(0)=\frac{\psi^{\prime \prime}(0)}{q}+\frac{2 \psi^{\prime}(0) \psi_{0}^{\prime}(0)}{q^{2}}=\frac{\sigma^{2}+a^{2}}{q}+\frac{2 a a_{0}}{q^{2}} .
$$

If we additionally use the relations

$$
\mathbf{E} S_{\nu}^{2}=\varphi^{\prime \prime}(0), \quad \mathbf{D} S_{\nu}=\mathbf{E} S_{\nu}^{2}-\frac{a^{2}}{q^{2}}
$$

then we get (8).

The following Corollary is very useful in applications (see [11], p.333)

Corrolary 2.1

Let under the conditions of Proposition 2.1 the quantity $a=\mathbf{E} \zeta<\infty$ is fixed and let

$$
q=\mathbf{P}(\epsilon=1) \rightarrow 0
$$

Then

$$
\lim _{q \rightarrow 0} \mathbf{P}\left(q S_{\nu}<t\right)=1-\exp \left(-\frac{t}{a}\right)
$$

Proof

If

$$
\psi_{1}(z)=\psi(z)-\psi_{0}(z)=\int_{0}^{\infty} \exp (-z t) d F_{\zeta, 1}(t)
$$

then

$$
\psi_{1}(0)=\mathbf{P}(\epsilon=1)=q .
$$

From the estimate

$$
1-\exp (-x) \leq x, \quad x>0
$$

we get

$$
\begin{gathered}
\psi_{1}(0)-\psi_{1}(q z)=\int_{0}^{\infty}(1-\exp (-q z t)) d F_{\zeta, 1}(t) \leq q z \int_{0}^{\infty} t d F_{\zeta, 1}(t) \\
\leq q z\left[K \int_{0}^{K} d F_{\zeta, 1}(t)+\int_{K}^{\infty} t d F_{\zeta, 1}(t)\right] \leq q z[K q+\mathbf{E} \zeta I(\zeta>K) I(\epsilon=1)] .
\end{gathered}
$$

Inserting $K=\frac{1}{\sqrt{q}}$, we get

$$
0 \leq \psi_{1}(0)-\psi_{1}(q z) \leq q z(\sqrt{q}+o(1))
$$


That is why

$$
\lim _{q \rightarrow 0} \frac{\psi_{1}(q z)}{q}=1
$$

uniformly on $z \in(0, C), \forall C>0$.

Making use of the relations

$$
\mathbf{P}\left(q S_{\nu}<t\right)=F_{S}\left(\frac{t}{q}\right), \quad \int_{0}^{\infty} \exp (-z t) d F_{S}\left(\frac{t}{q}\right)=\varphi(q z),
$$

and the convergence of the Laplace transform ([6], p.431), we come to conclusion that in order to prove equality (12) it is sufficient to show that

$$
\lim _{q \rightarrow 0} \varphi(q z)=\frac{1}{1+a z} .
$$

We have

$$
\varphi(q z)=\frac{\psi(q z)-\psi_{0}(q z)}{1-\psi_{0}(q z)}=\frac{\psi_{1}(q z) / q}{(1-\psi(q z)) / q+\psi_{1}(q z) / q} .
$$

From relation

$$
\lim _{q \rightarrow 0} \frac{(1-\psi(q z))}{q z}=-\psi^{\prime}(0)=a
$$

and relations (13) and (15), relation (14) follows.

Remark 2.1. In the recent paper [24] the limit theorems for some regenerative processes were proved based on asymptotic relations of the (12) type. The asymptotic relations (12) can be applied when investigating the length of the queue in stochastic networks (see, for example, [15], [7], [8]).

\section{Corrolary 2.2}

Let $\tau$ and $\eta$ be independent random variables, let

$$
\begin{gathered}
F(t)=\mathbf{P}(\tau<t)=1-\exp (-\lambda t), t \geq 0, \quad G(t)=\mathbf{P}(\eta<t), \quad G(+0)=0, \\
\epsilon=I(\tau<\eta), \quad \mathbf{P}(\epsilon=1)=q, \quad 0<q<1 .
\end{gathered}
$$

Denote by $\left(\tau_{i}, \eta_{i}, \epsilon_{i}\right)$ a sequence of independent copies of $(\tau, \eta, \epsilon)$,

$$
\begin{gathered}
\nu=\min \left(n \geq 1: \quad \epsilon_{n}=1\right), \\
S_{\nu}=\sum_{i=1}^{\nu} \min \left(\tau_{i}, \eta_{i}\right) .
\end{gathered}
$$

Then

$$
P_{S}(t)=\mathbf{P}\left(S_{\nu} \geq t\right)=\exp (-\lambda t)
$$

Proof

If we take $\zeta=\min (\tau, \eta)$, then in notations of Proposition 2.1 we have

$$
\begin{gathered}
1-F_{\zeta}(t)=\mathbf{P}(\min (\tau, \eta) \geq t)=\exp (-\lambda t)(1-G(t)), \\
F_{\zeta, 0}(t)=\mathbf{P}(\min (\tau, \eta)<t, \tau \geq \eta)=\int_{0}^{t} \exp (-\lambda x) d G(x) .
\end{gathered}
$$

Substituting these functions into equation (5) we get

$$
P_{S}(t)=\exp (-\lambda t)(1-G(t))+\int_{0}^{t} P_{S}(t-x) \exp (-\lambda x) d G(x) .
$$


By Proposition 2.1 the Laplace transform of the function $F_{S}(t)=1-P_{S}(t)$ is represented by formula (6). We have to find the functions $\psi(z)$ and $\psi_{0}(z)$.

Take $\phi(z)=\int_{0}^{\infty} \exp (-z t) d G(t)$. Then

$$
\psi_{0}(z)=\int_{0}^{\infty} \exp (-z t) \exp (-\lambda t) d G(t)=\phi(z+\lambda),
$$

and

$$
\begin{gathered}
\psi(z)=\int_{0}^{\infty} \exp (-z t) d(-\exp (-\lambda t)(1-G(t)) \\
=\lambda \int_{0}^{\infty} \exp (-(z+\lambda) t)(1-G(t)) d t+\int_{0}^{\infty} \exp (-(z+\lambda) t) d G(t) \\
=\frac{\lambda}{z+\lambda}-\lambda \int_{0}^{\infty} \exp (-(z+\lambda) t) G(t) d t+\phi(z+\lambda) \\
=\frac{\lambda}{z+\lambda}(1-\phi(z+\lambda))+\phi(z+\lambda) .
\end{gathered}
$$

Making use of the functions $\psi(z)$ and $\psi_{0}(z)$ from the formula (6), we get

$$
\varphi(z)=\frac{\lambda}{z+\lambda}
$$

This means that equality (16) is correct.

It is easy to make sure that substitution $P_{S}(t)=\exp (-\lambda t)$ in equation (17) converts it into identity.

Remark 2.2. If under conditions of Proposition 2.1 the random variables $\epsilon$ and $\zeta$ are independent, then

$$
F_{\zeta, 0}(t)=(1-q) F_{\zeta}(t), \quad \psi_{0}(z)=(1-q) \psi(z)
$$

and equality (6) is of the form

$$
\varphi(z)=\frac{q \psi(z)}{1-(1-q) \psi(z)} .
$$

Ofcourse, under this condition, equality (18) can be deduced directly from the definition.

If, additionally, the random variable $\zeta$ has the exponential distribution, $F_{\zeta}(t)=1-\exp (-\lambda t), t \geq 0$, then

$$
\psi(z)=\frac{\lambda}{z+\lambda}, \quad \varphi(z)=\frac{q \lambda}{z+q \lambda} .
$$

The last equality means that the random variable $S_{\nu}$ has the exponential distribution with parameter $\lambda q$,

$$
F_{S}(t)=1-\exp (-\lambda q t), t \geq 0 .
$$

Remark 2.3. For independent random variables $\nu$ and $\zeta$ in the book [1] the following formula is proposed

$$
\mathbf{D} S_{\nu}=\mathbf{D} \zeta \mathbf{E} \nu+(\mathbf{E} \zeta)^{2} \mathbf{D} \nu
$$

Unfortunately, as follows from Proposition 2.1, for arbitrary Markov moments this simple formula (19) is incorrect. In general case similar formulas are known only for the values $\mathbf{E}\left(S_{\nu}-\nu \mathbf{E} \zeta\right)^{2}$ (see books [1], [16]) 


\section{Applications}

\subsection{Application 1. Geiger-Muller counter of type 1.}

Such a counter is used to calculate cosmic particles which have arrived in some area of space. It acts as follows ([22], p.273, [6], p.372). A particle reaching the counter when it is free is registered but locks the counter for a random time $\eta$. Particles reaching the counter during the locked period are not registered. Denote by $T$ the time during which the Geiger-Muller counter will not lose any particles, and let $P_{T}(t)=\mathbf{P}(T>t)$.

To simplify recordings, we assume that at the moment $t_{0}=0$ a particle reaching the counter, and $t_{1}, t_{2}, \ldots, t_{k}, \ldots$ are the next moments of particle reaching, and denote $\tau_{k}=t_{k}-t_{k-1}$.

Let $\eta_{k}$ be the discharge time of the particle reaching the counter at the moment $t_{k-1}$, and let $\left(\tau_{k}\right)$ and $\left(\eta_{k}\right)$ be independent random sequences with continuous distribution functions,

$$
\mathbf{P}\left(\tau_{k}<t\right)=F(t), \quad \mathbf{P}\left(\eta_{k}<t\right)=G(t), \quad F(+0)=0, \quad G(+0)=0,
$$

and

Then it is clear that

$$
\epsilon_{k}=I\left(\tau_{k}<\eta_{k}\right), k \geq 1, \quad \nu=\min \left(k \geq 1: \quad \epsilon_{k}=1\right) .
$$

$$
T=\sum_{i=1}^{\nu} \tau_{i} .
$$

In order to apply Proposition 2.1 here, we take

$$
\begin{gathered}
\zeta_{i}=\tau_{i}, \quad i \geq 1, \quad F_{\zeta}(t)=F(t), \\
F_{\zeta, 0}(t)=\mathbf{P}\left(\tau_{1}<t, \tau_{1}>\eta_{1}\right)=\int_{0}^{t} G(x) d F(x), \\
q=\mathbf{P}\left(\tau_{1}<\eta_{1}\right)=\int_{0}^{\infty} F(t) d G(t) .
\end{gathered}
$$

The equality (5) in this case is of the form

$$
P_{T}(t)=1-F(t)+\int_{0}^{t} P_{T}(t-x) G(x) d F(x) .
$$

The Laplace transform $\varphi(z)$ for distribution of the random variable $T$ is determined by formula (6), in which

$$
\psi(z)=\int_{0}^{\infty} \exp (-z t) d F(t), \quad \psi_{0}(z)=\int_{0}^{\infty} \exp (-z t) G(t) d F(t) .
$$

The mean value of $\mathbf{E} T$ and the variance $\mathbf{D} T$ can be calculated by formulas (7) and (8).

In the most important case when there is a Poisson flow of particles with parameter $\lambda$,

$$
F(t)=1-\exp (-\lambda t), t \geq 0,
$$

we have

$$
\psi(z)=\frac{\lambda}{z+\lambda}, \quad \psi_{0}(z)=\frac{\lambda \phi(z+\lambda)}{z+\lambda}, \quad \varphi(z)=\frac{\lambda(1-\phi(z+\lambda))}{z+\lambda(1-\phi(z+\lambda))},
$$

where

$$
\phi(z)=\int_{0}^{\infty} \exp (-z t) d G(t)
$$

It follows from (3), (7) and (8) that

$$
\mathbf{E} T=\frac{1}{\lambda q}, \quad \mathbf{D} T=\frac{2\left(q+\lambda a_{0}\right)-1}{\lambda^{2} q^{2}},
$$

where

$$
q=1-\phi(\lambda), \quad a_{0}=\lambda \int_{0}^{\infty} t \exp (-\lambda t) G(t) d t
$$




\subsection{Application 2. Redundant system with renewal.}

The problem of reliability of redundant systems with renewal is devoted to a fairly large number of works, among which we note the works [11], [17], [18]. Here we consider a simple renewal case, namely, duplication, when each operating unit is associated with a single standby unit, which replaces the operating unit in case the latter fails. The element that failed is repaired, and after repair its characteristics equivalent to the original characteristics. Suppose that there is one repair unit in the system. In addition, we consider that the standby unit is in a partially energized state until it is connected instead of the primary unit (light standby). During the period it is in standby, it can fail but the probability of this is less than the same probability for the basic unit ( [11], p. 324-330). This is redundant system of type $(i i i / 2 / 1)$ in terms of the paper [4]. Denote by $\xi(t)$ the total number of defective units in the system at a moment $t$. We assume that $\xi(0)=0$ almost surely and say that the system is in a state $k$ at a moment $t$, if $\xi(t)=k, k=0,1,2$. A busy period is understood as a continuous period when the system is functioning properly (at least one element is functioning). Every busy period is followed by an idle period when both units fail.

We will assume that the failure-free time of the primary unit $\tau$, and the failure-free time of the standby unit $\tau^{\prime}$ have exponential distributions

$$
\mathbf{P}(\tau<t)=1-e^{-\lambda t}, \quad \mathbf{P}\left(\tau^{\prime}<t\right)=1-e^{-\lambda^{\prime} t}, \quad t \geq 0,
$$

while the renewal time (repairing time) $\eta$ has an arbitrary distribution $G(t)=\mathbf{P}(\eta<t), \quad G(0+)=0$.

We suppose that $\tau, \tau^{\prime}$ and $\eta$ are independent random variables. Let

$$
q=\mathbf{P}(\tau<\eta)=1-\int_{0}^{\infty} e^{-\lambda x} d G(x) .
$$

Denote by $W_{k}$ the k-th busy period. When the system is in the state 2 then the system fails, so $W_{1}$ is the time to first fail. For $k$-th busy period we have simple formulas for the first moments ([11], [4] )

$$
\mathbf{E} W_{1}=\frac{1}{\lambda}+\frac{1}{\left(\lambda+\lambda^{\prime}\right) q} \quad \mathbf{E} W_{k}=\frac{\lambda+q \lambda^{\prime}}{\lambda\left(\lambda+\lambda^{\prime}\right) q}, \quad k \geq 2 .
$$

Based on Proposition 2.1 we can deduce exact formulas for calculation the variation $\mathbf{D} W_{k}$. Really, let $\left(\tau_{i}, \tau_{i}^{\prime}, \eta_{i}\right)$ be independent copies of $\left(\tau, \tau^{\prime}, \eta\right)$, let

$$
\epsilon_{i}=I\left(\tau_{i}<\eta_{i}\right), i \geq 1, \quad \nu=\min \left(i \geq 1: \quad \epsilon_{i}=1\right) .
$$

In the paper [4] we can find the representation

$$
W_{1} \stackrel{d}{=} \sum_{i=1}^{\nu} \zeta_{i}
$$

where $\xi_{1} \stackrel{d}{=} \xi_{2}$ means equality of distributions of the random variables $\xi_{1}$ and $\xi_{2}$.

In this formula $\zeta_{i}=\min \left(\tau_{i}, \eta_{i}\right)+\tilde{\tau}_{i}$, random variables $\tilde{\tau}_{i}$ do not depend on $\tau_{i}$ and $\eta_{i}$, and so do not depend on $\nu$, $\mathbf{P}\left(\tilde{\tau}_{i}<t\right)=1-e^{-\left(\lambda+\lambda^{\prime}\right) t}, t \geq 0$.

Then by formula (8)

$$
\mathbf{D} W_{1}=\frac{\sigma^{2}}{q}+\frac{a^{2}(q-1)+2 a a_{0}}{q^{2}},
$$

where

$$
\begin{gathered}
a=\mathbf{E} \min (\tau, \eta)+\mathbf{E} \tilde{\tau}=\frac{q}{\lambda}+\frac{1}{\left(\lambda+\lambda^{\prime}\right)} \\
\sigma^{2}=\mathbf{D} \min (\tau, \eta)+\mathbf{D} \tilde{\tau}=\mathbf{E} \min (\tau, \eta)^{2}-\frac{q^{2}}{\lambda^{2}}+\frac{1}{\left(\lambda+\lambda^{\prime}\right)^{2}}
\end{gathered}
$$


For $k \geq 2$

$$
\begin{gathered}
=\frac{2-q^{2}}{\lambda^{2}}+\frac{1}{\left(\lambda+\lambda^{\prime}\right)^{2}}-2 \int_{0}^{\infty} t e^{-\lambda t} G(t) d t \\
a_{0}=\mathbf{E}[\min (\tau, \eta)+\tilde{\tau}] I(\tau>\eta)=\int_{0}^{\infty} t e^{-\lambda t} d G(t)+\frac{1-q}{\left(\lambda+\lambda^{\prime}\right)} .
\end{gathered}
$$

$$
W_{1} \stackrel{d}{=} W_{k}+\tilde{\tau},
$$

(see [4]), moreover $W_{k}, \tilde{\tau}$ are independent. That is why

$$
\mathbf{D} W_{k}=\mathbf{D} W_{1}-\frac{1}{\left(\lambda+\lambda^{\prime}\right)^{2}} .
$$

B. V. Gnedenko [9], [10] found an integral equation which the function $\mathbf{P}\left(W_{1}>t\right)$ satisfies, and derived a formula for the Laplace transform of this function. Unfortunately, under the general assumptions concerning the distribution $G(t)$, the explicit form of a function $\mathbf{P}\left(W_{1}>t\right)$ is not known. In the case $G(t)=1-e^{-\mu t}, t \geq 0$, the function $\mathbf{P}\left(W_{1}>t\right)$ is known (see, for example, [12]), but has a rather cumbersome form. Therefore, the following equations seem rather unexpected.

Consider the first busy period and denote by $\alpha_{0}$ and $\alpha_{1}$ times of stay of the system in the states 0 and 1 correspondingly. Therefore

$$
W_{1}=\alpha_{0}+\alpha_{1},
$$

moreover (see [4])

$$
\alpha_{0} \stackrel{d}{=} \sum_{i=1}^{\nu} \tilde{\tau}_{i}, \quad \alpha_{1} \stackrel{d}{=} \sum_{i=1}^{\nu} \min \left(\tau_{i}, \eta_{i}\right) .
$$

These representations together with the Corollary 2.2 of Proposition 2.1 and Remark 2.2 give the following equalities:

$$
\mathbf{P}\left(\alpha_{0}>t\right)=e^{-\left(\lambda+\lambda^{\prime}\right) q t}, \quad \mathbf{P}\left(\alpha_{1}>t\right)=e^{-\lambda t}, \quad t \geq 0 .
$$

It is clear that random variables $\alpha_{0}$ and $\alpha_{1}$ are strongly dependent, which does not allow to find a simple formula for distribution $\mathbf{P}\left(W_{1}>t\right)$.

\subsection{Application 3. Single-server queueing system}

Consider a single-server queueing system (SSQS) on the interval $[0, \infty)$. Let $t_{0}=0, t_{1}, \ldots, t_{n}, \ldots$ be the customer random arrival times. The arrived customer starts to be served, if the service channel is not occupied. Otherwise, the customer joins a queue whose size is not limited. The channel serves the $n$-th customer during a random time $\eta_{n}$. After the end of the service, the channel or takes the next customer, if the queue is not empty, or is waiting for a new customer.

Suppose that $\tau_{n-1}=t_{n}-t_{n-1}, n \geq 1$, are independent identically distributed random variables with the distribution function $F(t)=\mathbf{P}\left(\tau_{n}<t\right)=1-e^{-\lambda t}, t \geq 0$. It means that there is a Poisson frow of customer arrives to SSQS system (see [12]). Let $\eta_{n}, n \geq 1$, be independent identically distributed random variables with the distribution function $G(t)=\mathbf{P}\left(\eta_{n}<t\right), G(+0)=0$.

Denote by $\xi(t)$ the number of customers in the SSQS system at a moment of time $t, \xi(0)=0$ a.s. We say that at a moment $t$ SSQS system is in the state $k$, if $\xi(t)=k$.

We define the regeneration moments $S_{k}$ for the process $\xi(t)$ in the following way.

Let $S_{0}=t_{1}$ be the moment when the first customer arrives. Let $S_{1}$ be the first transition moment from the state 2 to the state 1 .

For $k>1$ the beginning of $k$-the regeneration cycle $S_{k-1}$ we take $k-1$-th transition moment from the state 2 to the state 1 while its end $S_{k}$ is $k$-th such a moment.

Denote, as in the previous application,

$$
\epsilon_{i}=I\left(\tau_{i}<\eta_{i}\right), i \geq 1, \quad \nu=\min \left(i \geq 1: \quad \epsilon_{i}=1\right) .
$$

Figure 1 demonstrates the first regeneration cycle. 


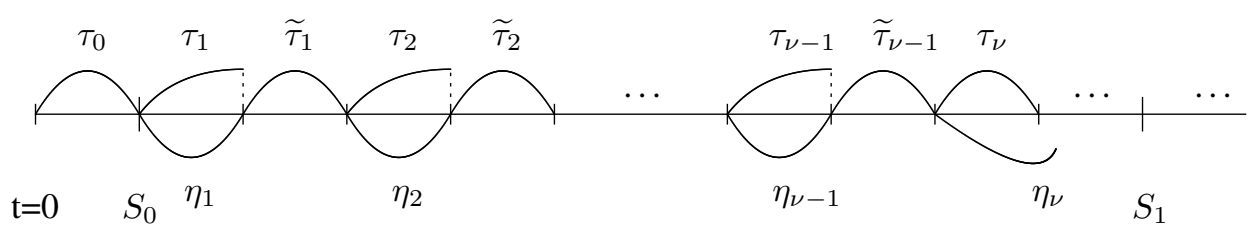

Figure 1.

The random variables $\widetilde{\tau}_{i}$ on Figure 1 do not depend on $\tau_{i}$ and $\eta_{i}$, and $\mathbf{P}\left(\widetilde{\tau}_{i}<t\right)=1-e^{-\lambda t}$.

We describe the work of the SSQS in the first period of regeneration. Let $T=S_{1}-S_{0}$ be its duration, let $S_{0}+\alpha$ be the first transition moment to the state 2 . On the interval $\left[S_{0}, S_{0}+\alpha\right)$ the system can only be in states 0 or 1 , the queue is absent. Next is the interval $\left[S_{0}+\alpha, S_{0}+\alpha+\beta\right)$ of length $\beta$, on which the queue is always $\geq 1$,

$$
T=\alpha+\beta .
$$

We find some basic characteristics of the random variables $\alpha$ and $\beta$.

Denote by $\alpha_{0}$ and $\alpha_{1}$ be sojourn times in states 0 and 1 on $\left[S_{0}, S_{1}\right)$. It is clear that

$$
\alpha=\alpha_{0}+\alpha_{1}
$$

We can find distributions of random variables $\alpha_{0}$ and $\alpha_{1}$ based on results of Proposition 2.1

$$
\begin{aligned}
& \mathbf{P}\left(\alpha_{0}>t\right)=(1-q) e^{-\lambda q t}, t>0, \\
& \mathbf{P}\left(\alpha_{0}=0\right)=q . \\
& \mathbf{P}\left(\alpha_{1}>t\right)=e^{-\lambda t}, t>0 .
\end{aligned}
$$

Proof of equality (26). We can conclude from Figure 1 that

$$
\alpha_{0}=\sum_{i=1}^{\nu-1} \widetilde{\tau}_{i}
$$

As we noted before the random variable $\nu$ and the sequence $\left(\widetilde{\tau}_{i}\right)$ are independent, $\widetilde{\tau}_{i}$ have the exponential distribution with parameter $\lambda$, that is why

$$
\mathbf{E} \exp \left(-z \alpha_{0}\right)=\sum_{k=1}^{\infty} q(1-q)^{k-1}\left[\mathbf{E} \exp \left(-z \widetilde{\tau}_{1}\right)\right]^{k-1}=\sum_{k=1}^{\infty} q\left(\frac{\lambda(1-q)}{z+\lambda}\right)^{k-1}=\frac{q(z+\lambda)}{z+q \lambda} .
$$

It is also not difficult to check that the distribution function

$$
H(t)=\left\{\begin{array}{rr}
0, & t<0 \\
1-(1-q) \exp (-\lambda q t), & t>0
\end{array}\right.
$$

satisfies the equality

$$
\int_{0}^{\infty} \exp (-z t) d H(t)=\frac{q(z+\lambda)}{z+q \lambda} .
$$

This means that equality (26) is correct.

Let's move to equality (27). It follows from Figure 1 that

$$
\alpha_{1}=\sum_{i=1}^{\nu} \min \left(\tau_{i}, \eta_{i}\right)
$$


So (27) follows from Corollary 2.1 of Proposition 2.1.

In the following we will assume that

$$
b=\mathbf{E} \eta_{i}<\infty, \quad \rho=\lambda b<1 .
$$

It is known [12] that in this case there exist stationary probabilities of states

$$
\lim _{t \rightarrow \infty} \mathbf{P}(\xi(t)=i)=p_{i}, \quad i=0,1,2, \ldots,
$$

and

$$
p_{0}=1-\rho \text {. }
$$

From (26), (27) we get

$$
\mathbf{E} \alpha_{0}=\frac{1-q}{\lambda q}, \quad \mathbf{E} \alpha_{1}=\frac{1}{\lambda}
$$

We need also equalities

$$
p_{0}=\frac{\mathbf{E} \alpha_{0}}{\mathbf{E} T}, \quad p_{1}=\frac{\mathbf{E} \alpha_{1}}{\mathbf{E} T}
$$

(see [4]).

From equalities (24), (28) - (30) it follows that

$$
\begin{gathered}
\mathbf{E} \alpha=\mathbf{E} \alpha_{0}+\mathbf{E} \alpha_{1}=\frac{1}{\lambda q}, \\
\mathbf{E} T=\frac{\mathbf{E} \alpha_{0}}{p_{0}}=\frac{1-q}{\lambda q(1-\rho)}, \\
\mathbf{E} \beta=\frac{\rho-q}{\lambda q(1-\rho)} .
\end{gathered}
$$

We also note that from the derived equations the following simple formula follows

$$
p_{1}=\frac{q(1-\rho)}{1-q} .
$$

\section{Conclusions}

We propose results of the investigation of properties of the random sums of random variables under the condition that the random summands (i.i.d. random variables) are not independent on the (random) number of summands. We consider the case, where the number of summands is the first moment of an event occurrence. We propose integral equation and some relations that determine distributions of random sums, their Laplace transforms and main characteristics (first and second moments). Some applications of the obtained results are described. We, first, analyse the distribution function of the time during which the Geiger-Muller counter of type 1 does not lose any particles. Then, the distribution function and its main characteristics of the busy period of a redundant system with renewal is nalysed. And, third, the distribution function of the sojourn times of a single-server queueing system is analysed.

\section{REFERENCES}

1. A. A. Borovkov, Probability theory, London: Universitext, Springer, 2013.

2. Y. S. Chow, H. Robbins, and D. Siegmund, Great expectations: The theory of optimal stopping, Boston etc.: Houghton Mifflin Company. XII, 1971.

3. D. R. Cox, Renewal theory, Methuen \& Co. Ltd., London; John Wiley \& Sons, Inc., New York, 1962. 
4. B.V. Dovgai, and I.K. Matsak, On a redundant system with renewals, Theory of Probability and Mathematical Statistics, Vol. 94, p. 63-76, 2017

5. W. Feller, An introduction to probability theory and its applications. Vol. 1. 2nd ed., New York etc.: John Wiley and Sons, Inc., 1968.

6. W. Feller, An introduction to probability theory and its applications. Vol. 2. 2nd ed., New York etc.: John Wiley and Sons, Inc., 1971.

7. V.K. Gedam, and S. B. Pathare, Estimation approaches for mean response time of a two stage open queueing network model, Statistics, Optimization \& Information Computing, Vol. 3, pp. 249-258, 2015.

8. V. K. Gedam, and S. B. Pathare, Some estimation approaches of intensities for a two stage open queueing network, Statistics Optimization and Information Computing. Vol. 2, No.1. pp. 33-46, 2014

9. B. V. Gnedenko, On a two-unit redundant system, Izv. Akad. Nauk SSSR, Tekh. Kibern., No. 4, p. 3-12, 1964 (In Russian).

10. B. V. Gnedenko, Doubling with renewal, Izv. Akad. Nauk SSSR, Tekh. Kibern., No. 5, p. 111-118. 1964 (In Russian).

11. B. V. Gnedenko, Yu. K. Belyayev, and A. D. Solovyev, Mathematical methods of reliability theory, New York - London: Academic Press, 1969.

12. B. V. Gnedenko, and I. N. Kovalenko, Introduction to queuing theory, Moskva: Nauka, 1966 (In Russian).

13. A. Gut, Stopped random walks. Limit theorems and applications. 2nd ed., New York, NY: Springer, 2009.

14. V. Kalashnikov, Geometric sums: bounds for rare events with applications, Amsterdam: Springer, 1997.

15. L. Kleinrock, Queueing systems.Computer application. Vol.2, New York: Wiley, 1976.

16. A. N. Kolmogorov, Selected works by A. N. Kolmogorov. Vol. II: Probability theory and mathematical statistics. Ed. by A. N. Shiryayev, Mathematics and Its Applications. Soviet Series. 26. Dordrecht etc.: Kluwer Academic Publishers, 1992.

17. V. S. Korolyuk, Stochastic models of systems, Kyiv: Naukova Dumka, 1989 (In Russian).

18. I. N. Kovalenko, Studies in the reliability analysis of complex systems, Kyiv: Naukova Dumka, 1975 (In Russian).

19. V. M. Kruglov, and V. Yu. Korolev, Limit theorems for random sums, Moskva: Izdatelstvo Moskovskogo Universiteta, 1990 (In Russian).

20. I. K. Matsak, On a single-server queueing system with refusal, Theory of Probability and Mathematical Statistics, Vol. 90, P. 153-160, 2015.

21. S. I. Resnick, Adventures in stochastic processes, Boston etc.: Birkhäuser, 2002.

22. W. L. Smith, Renewal theory and its ramifications, J. Roy. Statist. Soc. Ser. B 20, pp. 243-302, 1958.

23. A. Wald, Sequential analysis, New York: Wiley \& Sons, 1947.

24. O. K. Zakusylo, and I. K. Matsak, On extreme values of some regenerative processes, Theory of Probability and Mathematical Statistics, Vol. 97, pp. 57-71, 2018. 